\title{
Some notes on the paper "The equivalence of cone metric spaces and metric spaces"
}

\author{
Mehdi Asadi ${ }^{1 *}$, Billy E Rhoades ${ }^{2}$ and Hossein Soleimani ${ }^{3}$
}

\author{
* Correspondence: masadi@azu.ac. \\ ir \\ 'Department of Mathematics, \\ Zanjan Branch, Islamic Azad \\ University, Zanjan, Iran \\ Full list of author information is \\ available at the end of the article
}

\section{Abstract}

In this article, we shall show that the metrics defined by Feng and Mao, and Du are equivalent. We also provide some examples for one of the metrics.

\section{Introduction and preliminary}

Let $E$ be a topological vector space (t.v.s.) with zero vector $\theta$. A nonempty subset $K$ of $E$ is called a convex cone if $K+K \subseteq K$ and $\lambda K \subseteq K$ for each $\lambda \geq 0$. A convex cone $K$ is said to be pointed if $K \cap-K=\{\theta\}$. For a given cone $K \subseteq E$, we can define a partial ordering $\preccurlyeq$ with respect to $K$ by

$$
x \preccurlyeq y \Leftrightarrow y-x \in K .
$$

$x<y$ will stand for $x \leqslant y$ and $x \neq y$ while $x \ll y$ stands for $y-x \in K^{\circ}$, where $K^{\circ}$ denotes the interior of $K$. In the following, we shall always assume that $Y$ is a locally convex Hausdorff t.v.s. with zero vector $\theta, K$ is a proper, closed, and convex pointed cone in $Y$ with $K^{\circ} \neq \varnothing, e \in K^{\circ}$ and $\leqslant$ a partial ordering with respect to $K$. The nonlinear scalarization function $\xi_{e}: Y \rightarrow \mathbb{R}$ is defined by

$$
\xi_{e}(\gamma)=\inf \{r \in \mathbb{R}: y \in r e-K\}
$$

for all $y \in Y$.

We will use $P$ instead of $K$ when $E$ is a real Banach spaces.

Lemma 1.1 [1] For each $r \in R$ and $y \in Y$, the following statements are satisfied:

(i) $\xi_{e}(y) \leq r \Leftrightarrow y \in r e-K$.

(ii) $\xi_{e}(y)>r \Leftrightarrow y \notin r e-K$.

(iii) $\xi_{e}(y) \geq r \Leftrightarrow y \notin r e-K^{\circ}$.

(iv) $\xi_{e}(y)<r \Leftrightarrow y \in r e-K^{\circ}$.

(v) $\xi_{e}($.$) is positively homogeneous and continuous on Y$.

(vi) $y_{1} \in y_{2}+K \Rightarrow \xi_{e}\left(y_{2}\right) \leq \xi_{e}\left(y_{1}\right)$

(vii) $\xi_{e}\left(y_{1}+y_{2}\right) \leq \xi_{e}\left(y_{1}\right)+\xi_{e}\left(y_{2}\right)$ for all $y_{1}, y_{2} \in Y$.

Definition 1.2 [1] Let $X$ be a nonempty set. A vector-valued function $d: X \times X \rightarrow Y$ is said to be a TVS-cone metric, if the following conditions hold:

(C1) $\theta \leqslant d(x, y)$ for all $x, y \in X$ and $d(x, y)=\theta$ iff $x=y$

(C2) $d(x, y)=d(y, x)$ for all $x, y \in X$

(C3) $d(x, y) \leqslant(x, z)+d(z, y)$ for all $x, y, z \in X$. 
The pair $(X, d)$ is then called a TVS-cone metric space.

Huang and Zhang [2] discuss the case in which $Y$ is a real Banach space and call a vector-valued function $d: X \times X \rightarrow Y$ a cone metric if $d$ satisfies (C1)-(C3). Clearly, a cone metric space, in the sense of Huang and Zhang, is a special case of a TVS-cone metric space.

In the following, some conclusions are listed.

Lemma 1.3 [3] Let $(X, D)$ be a cone metric space. Then

$$
d(x, y)=\inf _{\{u \in P \mid D(x, y) \preccurlyeq u\}}\|u\|, \quad x, y \in X
$$

is a metric on $X$.

Theorem 1.4 [3] The metric space $(X, d)$ is complete if and only if the cone metric space $(X, D)$ is complete .

Theorem 1.5 [1] Let $(X, D)$ be a TVS-cone metric space. Then $d_{2}: X \times X \rightarrow[0, \infty)$ defined by $d_{2}(x, y)=\xi_{e}(D(x, y))$ is a metric.

\section{Main results}

We first show that the metrics introduced the Lemma 1.3 and the Theorem 1.5 are equivalent. Then, we provide some examples involving the metric defined in Lemma 1.3 .

Theorem 2.1 For every cone metric $D: X \times X \rightarrow E$ there exists a metric $d: X \times X \rightarrow \mathbb{R}^{+}$which is equivalent to $D$ on $X$.

Proof. Define $d(x, y)=\inf \{\|u\|: D(x, y) \leqslant u\}$. By the Lemma $1.3 d$ is a metric. We shall now show that each sequence $\left\{x_{n}\right\} \subseteq X$ which converges to a point $x \in X$ in the $(X, d)$ metric also converges to $x$ in the $(X, D)$ metric, and conversely. We have

$$
\forall n, m \in \mathbb{N} \quad \exists u_{n m} \text { such that } \quad\left\|u_{n m}\right\|<d\left(x_{n}, x\right)+\frac{1}{m}, \quad D\left(x_{n}, x\right) \preccurlyeq u_{n m} .
$$

Put $v_{n}:=u_{n n}$ then $\left\|v_{n}\right\|<d\left(x_{n}, x\right)+\frac{1}{n}$ and $D\left(x_{n}, x\right) \leqslant v_{n}$. Now if $x_{n} \rightarrow x$ in $(X, d)$ then $d\left(x_{n}, x\right) \rightarrow 0$ and so $v_{n} \rightarrow 0$ too, therefore for all $c \gg 0$ there exists $N \in \mathbb{N}$ such that $v_{n} \prec \prec c$ for all $n \geq N$. This implies that $D\left(x_{n}, x\right) \prec \prec c$ for all $n \geq N$. Namely $x_{n} \rightarrow$ $x$ in $(X, D)$.

Conversely, for every real $\varepsilon>0$, choose $c \in E$ with $c \succ>0$ and $\|c\|<\varepsilon$. Then there exists $N \in \mathbb{N}$ such that $D\left(x_{n}, x\right) \prec \prec c$ for all $n \geq N$. This means that for all $\varepsilon>0$ there exists $N \in \mathbb{N}$ such that $d\left(x_{n}, x\right) \leq\|c\|<\varepsilon$ for all $n \geq N$. Therefore $d\left(x_{n}, x\right) \rightarrow 0$ as $n$ $\rightarrow \infty$ so $x_{n} \rightarrow x$ in $(X, d)$.

Theorem 2.2 If $d_{1}(x, y)=\inf \{\|u\|: D(x, y) \leqslant u\}$ and $d_{2}(x, y)=\xi_{e}(D(x, y))$ where $D$ is a cone metric on $X$. Then $d_{1}$ is equivalent with $d_{2}$.

Proof. Let $x_{n} \underset{d_{1}}{\rightarrow} x$ then $d_{1}\left(x_{n}, x\right) \underset{\mathbb{R}}{\rightarrow} 0$ so by Theorem 2.1 in $x_{n} \stackrel{D}{\rightarrow} x_{\text {so }}$

$$
\forall \varepsilon>0, \quad \forall e \succ \succ 0 \quad \exists N \quad \forall n\left(n \geq N \Rightarrow D\left(x_{n}, x\right) \prec \prec \varepsilon e\right),
$$

and or $\varepsilon e-D\left(x_{w}, x\right) \in K^{\circ}$ for all $n \geq N$. So $D\left(x_{w}, x\right) \in e-K^{\circ}$ for $n \geq N$. Now by [[1], Lemma 1.1 (iv)] $\xi_{e}\left(D\left(x_{n}, x\right)\right)<\varepsilon$ for all $n \geq N$. Namely $d_{2}\left(x_{n}, x\right)<\varepsilon$ for all $n \geq N$ therefore $d_{2}\left(x_{n}, x\right) \stackrel{\mathbb{R}}{\rightarrow} 0$ or $x_{n} \stackrel{d_{2}}{\rightarrow} x$. 
Conversely, $x_{n} \underset{d_{2}}{\rightarrow}$ hence $d_{2}\left(x_{n}, x\right) \underset{\mathbb{R}}{\rightarrow} 0$ so $\xi_{e}\left(D\left(x_{n}, x\right)\right) \underset{\mathbb{R}}{\rightarrow} 0$, therefore

$$
\forall \varepsilon>0 \quad \exists N \quad \forall n\left(n \geq N \Rightarrow \xi_{e}\left(D\left(x_{n}, x\right)\right)<\varepsilon\right) .
$$

So $D\left(x_{n}, x\right) \in \varepsilon e-K^{\circ}$ for $n \geq N$ by [[1], Lemma 1.1 (iv)]. Hence, $D\left(x_{n}, x\right)=\varepsilon e-k$ for some $k \in K^{\circ}$, so $D\left(x_{n}, x\right) \prec \prec \varepsilon e$ for $n \geq N$ this implies that $x_{n} \stackrel{D}{\rightarrow} x$ and again by Theo-

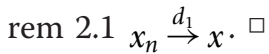

In the following examples, we use the metric of Lemma 1.3.

Example 2.3 Let $0 \neq a \in P \subseteq \mathbb{R}^{n}$ with $\|a\|=1$ and for every $x, y \in \mathbb{R}^{n}$ define

$$
D(x, y)= \begin{cases}a, & x \neq y \\ 0, & x=y\end{cases}
$$

Then $D$ is a cone metric on $\mathbb{R}^{n}$ and its equivalent metric $d$ is

$$
d(x, y)=\left\{\begin{array}{l}
1, x \neq y \\
0, x=y
\end{array}\right.
$$

which is a discrete metric.

Example 2.4 Let $a, b \geq 0$ and consider the cone metric $D: \mathbb{R} \times \mathbb{R} \rightarrow \mathbb{R}^{2}$ with

$$
D(x, y)=\left(a d_{1}(x, y), b d_{2}(x, y)\right)
$$

where $d_{1}, d_{2}$ are metrics on $\mathbb{R}$. Then its equivalent metric is

$$
d(x, y)=\sqrt{a^{2}+b^{2}}\left\|\left(d_{1}(x, y), d_{2}(x, y)\right)\right\| .
$$

In particular if $d_{1}(x, y):=|x-y|$ and $d_{2}(x, y):=\alpha|x-y|$, where $\alpha \geq 0$ then $D$ is the same famous cone metric which has been introduced in [[2], Example 1] and its equivalent metric is

$$
d(x, y)=\sqrt{1+\alpha^{2}}|x-y|
$$

Example 2.5 For $q>0, b>1, E=l^{q}, P=\left\{\left\{x_{n}\right\}_{n \geq 1}: x_{n} \geq 0\right.$, for all $\left.n\right\}$ and $(X, \rho) a$ metric space, define $D: X \times X \rightarrow E$ which is the same cone metric as [[4], Example 1.3] by

$$
D(x, y)=\left\{\left(\frac{\rho(x, y)}{b^{n}}\right)^{\frac{1}{q}}\right\}_{n \geq 1}
$$

Then its equivalent metric on $\times$ is

$$
d(x, y)=\left\|\left\{\left(\frac{\rho(x, y)}{b^{n}}\right)^{\frac{1}{q}}\right\}_{n \geq 1}\right\|_{l^{q}}=\left(\sum_{n=1}^{\infty} \frac{\rho(x, y)}{b^{n}}\right)^{\frac{1}{q}}=\left(\frac{\rho(x, y)}{b-1}\right)^{\frac{1}{q}} .
$$


Mansour Vaezpour for his helpful advise which led them to present this article. They also express their deep gratitude to the referee for his/her valuable comments and suggestions.

\section{Author details}

'Department of Mathematics, Zanjan Branch, Islamic Azad University, Zanjan, Iran ${ }^{2}$ Department of Mathematics, Indiana University, Bloomington, IN 47405-7106, USA ${ }^{3}$ Department of Mathematics, Malayer Branch, Islamic Azad University, Malayer, Iran

\section{Authors' contributions}

All authors have read and approved the final manuscript.

\section{Competing interests}

The authors declare that they have no competing interests.

Received: 9 May 2011 Accepted: 21 May 2012 Published: 21 May 2012

\section{References}

1. Du, WS: A note on cone metric fixed point theory and its equivalence. Nonlinear Anal. 72, 2259-2261 (2010). doi:10.1016/..na.2009.10.026

2. Huang, LG, Zhang, X: Cone metric spaces and fixed point theorems of contractive mapping. J Math Anal Appl. 322(2), 1468-1476 (2007)

3. Feng, Y, Mao, W: Equivalence of cone metric spaces and metric spaces. Fixed Point Theory. 11(2), 259-264 (2010)

4. Haghi, RH, Rezapour, Sh: Fixed points of multifunctions on regular cone metric spaces. Expositiones Mathematicae. 28(1), 71-77 (2010). doi:10.1016/j.exmath.2009.04.001

doi:10.1186/1687-1812-2012-87

Cite this article as: Asadi et al: Some notes on the paper "The equivalence of cone metric spaces and metric spaces". Fixed Point Theory and Applications 2012 2012:87.

\section{Submit your manuscript to a SpringerOpen ${ }^{\circ}$} journal and benefit from:

Convenient online submission

- Rigorous peer review

- Immediate publication on acceptance

- Open access: articles freely available online

- High visibility within the field

- Retaining the copyright to your article

Submit your next manuscript at $\gg$ springeropen.com 